\title{
Development of an Enteric Bacterial Enrichment Broth and Its Performance for Isolation of Clinically Significant Bacterial Pathogens from Stool
}

\author{
Timothy S. Horseman*, Michael B. Lustik, Keith S. K. Fong \\ Department of Clinical Investigation, Tripler Army Medical Center, Honolulu, USA \\ Email: ^timothy.s.horseman.mil@mail.mil
}

How to cite this paper: Horseman, T.S., Lustik, M.B. and Fong, K.S.K. (2021) Development of an Enteric Bacterial Enrichment Broth and Its Performance for Isolation of Clinically Significant Bacterial Pathogens from Stool. Open Journal of Medical Microbiology, 11, 1-17.

https://doi.org/10.4236/ojmm.2021.111001

Received: December 2, 2020

Accepted: January 31, 2021

Published: February 3, 2021

Copyright $\odot 2021$ by author(s) and Scientific Research Publishing Inc. This work is licensed under the Creative Commons Attribution International License (CC BY 4.0).

http://creativecommons.org/licenses/by/4.0/

\begin{abstract}
Background: Early detection and accurate identification of foodborne pathogen outbreaks is an important public health function. Increased clinical adoption of multiplex PCR assays or culture independent diagnostic tests (CIDT) correlates to more stool specimens sent to public health laboratories (PHL) for characterization. Isolation and confirmation of enteric bacterial pathogens can prove difficult to consistently recover. The purpose of this study was to evaluate the performance of a broad-use laboratory developed enrichment broth for isolation of Campylobacter, Salmonella, Shigella, and Yersinia strains from stool specimens. Methods: The study compared differences in positivity rates among media and enrichment combinations at specific time points. Comparison of direct inoculation (DI), enrichment using a lab-developed Enteric Bacterial Enrichment (EBE) broth and gold-standard isolation methods were conducted to test current utility of this established practice with stool specimens heat injured and non-injured. Results: A total of 234 spiked stool samples, 175 non-injured and 59 heat injured, were tested with varying bacterial concentrations. For non-injured stools, direct inoculation performed better for Campylobacter and Yersinia than enrichment. Conversely, Salmonella and Shigella recovery and limit of detection increased with enrichment. Campylobacter had the highest percent recovery while Shigella being the lowest from direct plating at 6-hour and 24-hour enrichment periods. Among broths, EBE performed the best for Yersinia and similar to Selenite broth for Salmonella and Shigella. Generally, heat injured stool had a significantly lower percent of recovery than non-heat injured with a higher limit of detection across organisms. Conclusion: Our data suggest there is an only utility for targeted enrichment of CIDT positive Salmonella stool specimens.
\end{abstract}


We highlight the difficulties of formulating an enrichment broth capable of supporting a variety of enteric pathogens with standardized incubation. Increasing demands on PHL infrastructure warrant further examination of enhancing organism isolation and cost analyses for CIDT positive specimens.

\section{Keywords}

Enteric Bacteria, Enrichment, Stool, Selective Agar

\section{Introduction}

Gastroenteritis is a major cause of morbidity worldwide, and clinical presentation alone is unable to distinguish organism etiologies. Early detection and accurate identification of gastrointestinal (GI) bacterial outbreaks is an important public health function. Historically stool culture was the main diagnostic method in clinical microbiology and public health laboratories (PHL) for the identification of GI bacterial pathogens. However, in recent years, multiplex molecular assays have been developed and widely implemented for the detection of GI pathogens directly from clinical stool specimens [1] [2]. These multiplex panels are rapid and sensitive assays although multiple organism detections can cloud interpretation [3]. Traditionally, PHL have received bacterial isolates for organism confirmation and further characterization to aid in disease surveillance. As more laboratories adopt multiplex assays or culture independent diagnostic tests (CIDT), positive stool specimens will be sent to PHL instead of isolates. Recovery and confirmation of these bacterial pathogens will fall to the surveillance laboratories accruing further costs [4] [5].

Gastrointestinal bacterial pathogens can prove difficult to consistently recover for a variety of reasons including transport time and temperature, competition with saprophytic gut flora, and the variance in incubation conditions for cultivation [2]. Tennessee Public Health Laboratory reported pathogen recovery was 57\% from referred CIDT-positive stool specimens, which varied greatly based on pathogen type with Salmonella (72\%) recovered the most frequently in contrast to Campylobacter (26\%) [6]. If a bacterial isolate cannot be recovered from a positive CIDT specimen, monitoring disease burden and enacting public health interventions could be significantly more difficult.

There have been many reports in the literature concerning the use of enrichment broths and selective agars for the isolation of GI bacteria [7] [8] [9] [10] [11]. Isolation of bacterial pathogens from food matrices using a variety of commercial and in-house developed selective media and enrichment methods is heavily documented [12] [13]. Unfortunately, the use of gold-standard media and enrichment broths in clinical microbiology has not significantly changed over the last 50 years [14] [15] [16] [17]. Well-performing chromogenic agars have been developed but are expensive for routine, high volume use, and are 
mainly pathogen specific [18]. Historically, broth enrichment has been used to increase detection of bacterial pathogens especially in times of extended sample transport to the laboratory. Although an overabundance of evidence points to the utility of enrichment, the cost-effectiveness and value of enrichment broths has been questioned [19]. The increased financial burden to PHL having to recover isolates from CIDT positive stool specimens eliminates the potential for pathogen-specific gold-standard enrichment to be used routinely. There is a need to re-evaluate bacterial isolation and screen for broad use methods to increase recovery of GI pathogens. A multi-pathogen broth with standardized incubation conditions, novel in clinical enteric bacterial recovery, can be economically beneficial since it reduces labor and testing cost per sample.

The purpose of this pilot study was to evaluate a broad-use laboratory developed enrichment broth, and to provide comparison of selective agars and enrichment combinations at specific time points for GI pathogen isolation.

\section{Materials and Methods}

\subsection{Bacterial Pathogens and Sample Preparation}

Negative clinical stool specimens from Tripler Army Medical Center (TAMC) were pooled and aliquots created for bacterial spiking of specimens. The following bacterial pathogens and number of strains were included in the study: Campylobacter $(\mathrm{n}=20)$, Salmonella $(\mathrm{n}=10)$, Shigella $(\mathrm{n}=6)$, and Yersinia $(\mathrm{n}=4)$. Bacteria were isolated from TAMC clinical stools or obtained from American Type Culture Collection (ATCC) (Table 1). Bacterial suspensions were prepared from fresh overnight culture growth to optical density of 0.5 McFarland $\left(\sim 10^{8}\right.$ $\mathrm{CFU} / \mathrm{mL}$ ). Approximately 0.5 gram negative stool was diluted into $8.5 \mathrm{~mL}$ sterile saline $(0.9 \% \mathrm{NaCl})$ and spiked with $1 \mathrm{~mL} 0.5 \mathrm{McFarland}$ Standard bacterial suspension to generate 10 -fold serial dilutions $\left(\sim 1 \times 10^{7}-10^{0} \mathrm{CFU} / \mathrm{mL}\right)$ in stool solution. Each stool dilution represented a unique specimen in an attempt to mimic receipt of different bacterial concentration burdens clinically. A stool suspension without bacteria was used as negative control and plated to confirm negative result.

\subsection{Culture Media and Enrichment Broths}

Bacteria were cultured on agar plates: Hektoen (HEK), Xylose-Lysine-Desoxycolate (XLD), Cefsulodin Irgasan Novobiocin (CIN) (BD Diagnostics, Franklin Lakes, NJ, USA), HardyCHROM SS NoPro agar and Modified Charcoal Cefoperazone Deoxycholate (mCCDA) (Hardy Diagnostics, Santa Maria, CA, USA). Commercial broths used were: GN broth, Selenite broth (SB) (BD Diagnostics, Franklin Lakes, NJ, USA), and EE Mossel broth (EE) (Teknova. Hollister, CA, USA). Results were interpreted based on manufacturer recommendations. Enteric Bacterial Enrichment (EBE) broth was prepared with ingredients from Sigma-Aldrich as follows: $30 \mathrm{~g}$ soybean-casein digest, $10 \mathrm{~g}$ meat extract, $0.6 \mathrm{~g} \mathrm{~L}$-cysteine, $1.0 \mathrm{~g}$ sodium pyruvate, $0.8 \mathrm{~g}$ potassium phosphate, $0.8 \mathrm{~g}$ sodium carbonate, $0.6 \mathrm{~g}$ bile 
salts, and $0.5 \mathrm{~g}$ sodium bisulfite per liter of distilled water (Sigma-Aldrich, St. Louis, MO, USA). After autoclaving, $100 \mu \mathrm{g} / \mathrm{ml}$ cycloheximide, $1 \mu \mathrm{g} / \mathrm{ml}$ rifampicin, and $30 \mathrm{mg}$ dissolved hemin were added to broth before use. EBE broth was modified from a successful Food Pathogen Enrichment broth developed to support growth of Campylobacter without lysed blood and $\mathrm{CO}_{2}$ [20].

Table 1. Genera and number of strains used to evaluate media and enrichment broths.

\begin{tabular}{|c|c|c|c|}
\hline Organism & Clinical $^{*}$ & Reference $^{\#}$ & Total \\
\hline Campylobacter sp. & 17 & 3 & 20 \\
\hline Campylobacter coli & 1 & 2 & 3 \\
\hline Campylobacter jejuni & 16 & 0 & 16 \\
\hline Campylobacter lari & 0 & 1 & 1 \\
\hline Salmonella sp. & 7 & 3 & 10 \\
\hline Salmonella Anatum & 0 & 1 & 1 \\
\hline Salmonella group B & 1 & 0 & 1 \\
\hline Salmonella group C1 & 1 & 0 & 1 \\
\hline Salmonella group C2 & 1 & 0 & 1 \\
\hline Salmonella group E & 1 & 0 & 1 \\
\hline Salmonella Paratyphi A & 1 & 1 & 2 \\
\hline Salmonella Typhumurium & 0 & 1 & 1 \\
\hline Non-typhoidal Salmonella, no group & 2 & 0 & 2 \\
\hline Shigella sp. & 2 & 4 & 6 \\
\hline Shigella boydii & 0 & 1 & 1 \\
\hline Shigella flexneri & 0 & 2 & 2 \\
\hline Shigella sonnei & 2 & 1 & 3 \\
\hline Yersinia sp. & 1 & 3 & 4 \\
\hline Yersinia enterocolitica & 1 & 1 & 2 \\
\hline Yersinia kristensenii & 0 & 1 & 1 \\
\hline Yersinia ruckeri & 0 & 1 & 1 \\
\hline
\end{tabular}

${ }^{*}$ Clinical strains were isolated from stool specimens; ${ }^{*}$ All reference strains were as follows: C. coli ATCC 33559; C. lari ATCC 35221; $S$. Anatum E9270; $S$. Paratyphi A ATCC 9150; $S$. Typhimurium ATCC 14028; $S$. boydii ATCC 9207; S. flexneri ATCC 9199; S. flexnerisero 2b ATCC 12022; S. sonnei ATCC 25931; Y. enterocolitica ATCC 9610; Y. kristensenii ATCC 33639; Y. ruckeri ATCC 29473. 


\subsection{Direct Inoculation and Subculture}

Serially diluted stools were inoculated directly onto each media type and into enrichment broth(s) with approximately $100 \mu \mathrm{L}$ or 2 drops. A thin-layer of mineral oil ( 3 - 5 drops) was added to each broth prior to incubation. Broths were incubated aerobically at $37^{\circ} \mathrm{C}$ for 24 hours. Enrichment broths were subcultured at 6 hours and 24 hours to the appropriate media types for each organism. HEK, $\mathrm{XLD}$, and NoPro SS plates were incubated aerobically at $37^{\circ} \mathrm{C}$ for $18-24$ hours, while mCCDA selective plates were incubated micro aerobically up to 72 hours at $42^{\circ} \mathrm{C}$. CIN for Yersinia isolation was incubated at room temperature up to 48 hours. After incubation, media were examined for characteristic pathogen growth.

To simulate dramatic temperature shifts during transport, spiked stool, after initial inoculation, was subjected to a heat shock for 10 minutes at $50^{\circ} \mathrm{C}$. Heat injured stools were directly plated, inoculated to enrichment broth, incubated and subcultured as described above.

\subsection{Bacterial Isolate Identification}

Colonies on selective media were subcultured for identification based on morphology and appearance on each media. Up to 3 colonies were selected for downstream identification using standard biochemical methods for Campylobacter (Gram stain, oxidase, catalase, and hippurate tests), and VITEK MS or VITEK 2 (bioMérieux, Durham, NC, USA). Data was based on whether a particular bacterial pathogen was present or not.

\subsection{Statistical Analysis}

Rates of detection were summarized for each pathogen and analysis method (direct plating vs. enrichment broth at 6 and 24 hours). Rates were calculated separately for original stools and heat injured stools. P-values were generated based on the following methods. Multivariable fixed effect logistic regression models, conditioning on isolate number and dilution factor, were used to evaluate detection rate differences among media, broths, and analysis method (direct plating v. enrichment). Models were run separately by heat injured status for each pathogen. McNemar's tests, which are commonly used to analyze paired dichotomous data, were performed to assess differences in positivity between 6 and 24 hour plating for each broth/media combination. Analyses were based on the paired replicates that were plated for 6 and 24 hours for each isolate. All analyses were conducted using SAS statistical software version 9.4 (SAS Institute, Cary, NC, USA).

\subsection{Ethics Approval}

This protocol was reviewed and approved by Tripler Army Medical Center Scientific Review Committee (Protocol No. TAMC 19N02) and Ethical Committee. The study met the criteria for Research Not Involving Human Subjects to obtain negative stool specimens from routine diagnostic procedures. 


\section{Results}

\subsection{Non-Heat Injured Stools}

A total of 175 spiked stool samples from 20 Campylobacter, 10 Salmonella, 6 Shigella, and 4 Yersinia isolates were analyzed (Table 1). Dilutions from $10^{5}$ to $10^{1} \mathrm{CFU} / \mathrm{mL}$ were inoculated and subcultured with the exception of Shigella spiked to $10^{2} \mathrm{CFU} / \mathrm{mL}$. Due to higher prevalence of clinical isolates seen at TAMC more stools were spiked with Campylobacter $(\mathrm{n}=98)$ than any other organism followed by Salmonella $(\mathrm{n}=43)$, Shigella $(\mathrm{n}=18)$ and Yersinia $(\mathrm{n}=$ 16). Bacterial recovery was compared for direct inoculation and after enrichment of stools, in terms of dilutions, media and broth types. We found that pathogen recovery differed among bacterial types, between direct plating versus enrichment, and at specific enrichment subculture times. Overall rates of positive growth for each media and broth combination at specific enrichment inoculation time points are indicated in Table 2.

Campylobacter isolation was more successful with direct inoculation than enrichment at both subculture time points $(92 \%, 73 \%$, and $66 \%$ recovery for DI, 6-hr, and 24-hr, respectively, $\mathrm{p}<0.001$, Figure 1 (a)). Direct inoculation recovered at least $90 \%$ of strains down to $10^{2} \mathrm{CFU} / \mathrm{mL}$ far better than isolation at 6-hour (70\%) or 24-hour (50\%) enrichment (Figure 2(a)). Enrichment required concentrations one to two orders of magnitude higher to achieve the same level of recovery as direct inoculation.

Salmonella strain isolation improved from direct inoculation with 6-hour enrichment and significantly after 24-hour enrichment $(32.6 \%, 38.6 \%$, and $64.7 \%$, respectively; $\mathrm{p}=0.100$ for 6 -hr vs. DI and $\mathrm{p}<0.001$ for 24 -hr vs. DI). The limit of detection was best for 24-hour enrichment, which showed $73 \%$ recovery at $10^{3} \mathrm{CFU} / \mathrm{mL}$ and $93 \%$ recovery at $10^{4} \mathrm{CFU} / \mathrm{mL}$. Isolation at $24 \mathrm{hr}$ was $30 \%-$ $35 \%$ greater than direct inoculation and 6-hour enrichment recovery at the same dilutions (Figure 2(a)). Among media for Salmonella, XLD performed better than HEK and SS NoPro ( $\mathrm{p}<0.001$ for both comparisons) for direct inoculation and enrichment (Figure 1(a)). Overall, the most successful enrichment broth for Salmonella isolation was SB (82\%) outperforming EBE (71\%), GN (59\%), and EE $(47 \%)$ ( $p<0.001$ for all pairwise combinations with SB except $\mathrm{p}=0.038$ for SB vs. EBE at $24 \mathrm{hr}$; Figure 3(a)).

Shigella strains had the lowest percent recovery with no difference in isolation between direct inoculation and enrichment $(14.8 \%, 12.5 \%$, and $14.8 \%$ for DI, 6-hr, and 24-hr, respectively). Shigella isolation from dilutions between $10^{2}-10^{4}$ $\mathrm{CFU} / \mathrm{mL}$ was largely unsuccessful, with recoveries less than $20 \%$. Isolation was better at $10^{5} \mathrm{CFU} / \mathrm{mL}$ (with 55\% recovery for direct inoculation, Figure 2(a)). Shigella isolate yield was not effective for any agar or enrichment broth although, SS NoPro recovery was considerably better than XLD and HEK after 24-hour enrichment (26\% for SS No Pro vs. $8 \%$ for XLD, $\mathrm{p}=0.001$, and $10 \%$ for HEK, $\mathrm{p}=0.002$; Figure 1(a)). 
Table 2. Recovery of Salmonella, Shigella, and Yersinia from non-heat injured and heat injured stools for different media and broth combinations following 6-hour and 24-hour enrichment.

\begin{tabular}{|c|c|c|c|c|c|c|c|c|c|}
\hline \multirow{3}{*}{ Salmonella } & \multicolumn{8}{|c|}{ Non-heat injured stools } & \multirow{3}{*}{ p-value } \\
\hline & \multicolumn{4}{|c|}{$6 \mathrm{hr}$} & \multicolumn{4}{|c|}{$24 \mathrm{hr}$} & \\
\hline & Total & Negative & Positive & \%Positive & Total & Negative & Positive & \%Positive & \\
\hline \multicolumn{10}{|l|}{ Broth/Media } \\
\hline EE/HEK & 43 & 32 & 11 & 26 & 43 & 25 & 18 & 42 & 0.016 \\
\hline EE/NOPRO & 43 & 35 & 8 & 19 & 43 & 22 & 21 & 49 & $<0.001$ \\
\hline $\mathrm{EE} / \mathrm{XLD}$ & 43 & 28 & 15 & 35 & 43 & 22 & 21 & 49 & 0.070 \\
\hline GN/HEK & 43 & 37 & 6 & 14 & 43 & 17 & 26 & 60 & $<0.001$ \\
\hline GN/NOPRO & 43 & 30 & 13 & 30 & 43 & 19 & 24 & 56 & 0.003 \\
\hline GN/XLD & 43 & 28 & 15 & 35 & 43 & 17 & 26 & 60 & 0.013 \\
\hline EBE/HEK & 43 & 32 & 11 & 26 & 43 & 11 & 32 & 74 & $<0.001$ \\
\hline EBE/NOPRO & 43 & 33 & 10 & 23 & 43 & 19 & 24 & 56 & $<0.001$ \\
\hline $\mathrm{EBE} / \mathrm{XLD}$ & 43 & 23 & 20 & 47 & 43 & 7 & 36 & 84 & $<0.001$ \\
\hline SB/HEK & 43 & 15 & 28 & 65 & 43 & 9 & 34 & 79 & 0.070 \\
\hline SB/NOPRO & 43 & 16 & 27 & 63 & 43 & 8 & 35 & 81 & 0.022 \\
\hline \multirow[t]{2}{*}{ SB/XLD } & 43 & 8 & 35 & 81 & 43 & 6 & 37 & 86 & 0.688 \\
\hline & \multicolumn{8}{|c|}{ Non-heat injured stools } & \\
\hline \multirow[t]{2}{*}{ Shigella } & \multicolumn{4}{|c|}{$6 \mathrm{hr}$} & \multicolumn{4}{|c|}{$24 \mathrm{hr}$} & p-value \\
\hline & Total & Negative & Positive & $\%$ Positive & Total & Negative & Positive & $\%$ Positive & \\
\hline \multicolumn{10}{|l|}{ Broth/Media } \\
\hline EE/HEK & 18 & 16 & 2 & 11 & 18 & 18 & 0 & 0 & 0.480 \\
\hline EE/NOPRO & 18 & 15 & 3 & 17 & 18 & 16 & 2 & 11 & 1.000 \\
\hline $\mathrm{EE} / \mathrm{XLD}$ & 18 & 15 & 3 & 17 & 18 & 18 & 0 & 0 & 0.248 \\
\hline GN/HEK & 18 & 17 & 1 & 6 & 18 & 15 & 3 & 17 & 0.500 \\
\hline GN/NOPRO & 18 & 16 & 2 & 11 & 18 & 13 & 5 & 28 & 0.250 \\
\hline GN/XLD & 18 & 18 & 0 & 0 & 18 & 18 & 0 & 0 & -- \\
\hline EBE/HEK & 18 & 15 & 3 & 17 & 18 & 18 & 0 & 0 & 0.248 \\
\hline EBE/NOPRO & 18 & 15 & 3 & 17 & 18 & 11 & 7 & 39 & 0.125 \\
\hline $\mathrm{EBE} / \mathrm{XLD}$ & 18 & 15 & 3 & 17 & 18 & 15 & 3 & 17 & 1.000 \\
\hline SB/HEK & 18 & 15 & 3 & 17 & 18 & 14 & 4 & 22 & 1.000 \\
\hline SB/NOPRO & 18 & 17 & 1 & 6 & 18 & 13 & 5 & 28 & 0.125 \\
\hline \multirow[t]{2}{*}{$\mathrm{SB} / \mathrm{XLD}$} & 18 & 15 & 3 & 17 & 18 & 15 & 3 & 17 & 1.000 \\
\hline & \multicolumn{8}{|c|}{ Non-heat injured stools } & \\
\hline \multirow[t]{2}{*}{ Yersinia } & \multicolumn{4}{|c|}{$6 \mathrm{hr}$} & \multicolumn{4}{|c|}{$24 \mathrm{hr}$} & $\mathrm{p}$-value \\
\hline & Total & Negative & Positive & \%Positive & Total & Negative & Positive & \%Positive & \\
\hline \multicolumn{10}{|l|}{ Broth/Media } \\
\hline $\mathrm{EE} / \mathrm{CIN}$ & 16 & 7 & 9 & 56 & 16 & 12 & 4 & 25 & 0.063 \\
\hline GN/CIN & 16 & 9 & 7 & 44 & 16 & 13 & 3 & 19 & 0.125 \\
\hline EBE/CIN & 16 & 3 & 13 & 81 & 16 & 4 & 12 & 75 & 1.000 \\
\hline $\mathrm{SB} / \mathrm{CIN}$ & 16 & 10 & 6 & 38 & 16 & 8 & 8 & 50 & 0.500 \\
\hline
\end{tabular}


Continued

\begin{tabular}{|c|c|c|c|c|c|c|c|c|c|}
\hline \multirow{3}{*}{ Salmonella } & \multicolumn{8}{|c|}{ Heat injured stools } & \multirow{3}{*}{ p-value } \\
\hline & \multicolumn{4}{|c|}{$6 \mathrm{hr}$} & \multicolumn{4}{|c|}{$24 \mathrm{hr}$} & \\
\hline & Total & Negative & Positive & \%Positive & Total & Negative & Positive & \%Positive & \\
\hline \multicolumn{10}{|l|}{ Broth/Media } \\
\hline EE/HEK & 16 & 12 & 4 & 25 & 16 & 11 & 5 & 31 & 1.000 \\
\hline EE/NOPRO & 16 & 12 & 4 & 25 & 16 & 13 & 3 & 19 & 1.000 \\
\hline EE/XLD & 16 & 12 & 4 & 25 & 16 & 8 & 8 & 50 & 0.125 \\
\hline GN/HEK & 16 & 12 & 4 & 25 & 16 & 6 & 10 & 63 & 0.031 \\
\hline GN/NOPRO & 16 & 11 & 5 & 31 & 16 & 7 & 9 & 56 & 0.219 \\
\hline GN/XLD & 16 & 11 & 5 & 31 & 16 & 1 & 15 & 94 & 0.002 \\
\hline EBE/HEK & 16 & 13 & 3 & 19 & 16 & 4 & 12 & 75 & 0.004 \\
\hline EBE/NOPRO & 16 & 15 & 1 & 6 & 16 & 8 & 8 & 50 & 0.016 \\
\hline EBE/XLD & 16 & 12 & 4 & 25 & 16 & 6 & 10 & 63 & 0.031 \\
\hline SB/HEK & 16 & 3 & 13 & 81 & 16 & 1 & 15 & 94 & 0.625 \\
\hline SB/NOPRO & 16 & 4 & 12 & 75 & 16 & 0 & 16 & 100 & 0.134 \\
\hline SB/XLD & 16 & 1 & 15 & 94 & 16 & 0 & 16 & 100 & 1.000 \\
\hline \multirow{3}{*}{ Shigella } & \multicolumn{8}{|c|}{ Heat injured stools } & \multirow{3}{*}{ p-value } \\
\hline & & & & & & & & & \\
\hline & Total & Negative & Positive & \%Positive & Total & Negative & Positive & \%Positive & \\
\hline \multicolumn{10}{|l|}{ Broth/Media } \\
\hline EE/HEK & 13 & 11 & 2 & 15 & 13 & 13 & 0 & 0 & 0.480 \\
\hline EE/NOPRO & 13 & 13 & 0 & 0 & 13 & 12 & 1 & 8 & 1.000 \\
\hline EE/XLD & 13 & 12 & 1 & 8 & 13 & 13 & 0 & 0 & 1.000 \\
\hline GN/HEK & 13 & 13 & 0 & 0 & 13 & 11 & 2 & 15 & 0.480 \\
\hline GN/NOPRO & 13 & 11 & 2 & 15 & 13 & 13 & 0 & 0 & 0.480 \\
\hline GN/XLD & 13 & 13 & 0 & 0 & 13 & 12 & 1 & 8 & 1.000 \\
\hline EBE/HEK & 13 & 12 & 1 & 8 & 13 & 13 & 0 & 0 & 1.000 \\
\hline EBE/NOPRO & 13 & 10 & 3 & 23 & 13 & 10 & 3 & 23 & 1.000 \\
\hline EBE/XLD & 13 & 12 & 1 & 8 & 13 & 13 & 0 & 0 & 1.000 \\
\hline SB/HEK & 13 & 12 & 1 & 8 & 13 & 12 & 1 & 8 & 1.000 \\
\hline SB/NOPRO & 13 & 12 & 1 & 8 & 13 & 12 & 1 & 8 & 1.000 \\
\hline SB/XLD & 13 & 11 & 2 & 15 & 13 & 11 & 2 & 15 & -- \\
\hline \multirow{3}{*}{ Yersinia } & \multicolumn{8}{|c|}{ Heat injured stools } & \multirow{3}{*}{ p-value } \\
\hline & & & & & & & & & \\
\hline & Total & Negative & Positive & $\%$ Positive & Total & Negative & Positive & \%Positive & \\
\hline \multicolumn{10}{|l|}{ Broth/Media } \\
\hline $\mathrm{EE} / \mathrm{CIN}$ & 10 & 4 & 6 & 60 & 10 & 8 & 2 & 20 & 0.125 \\
\hline GN/CIN & 10 & 8 & 2 & 20 & 10 & 8 & 2 & 20 & 1.000 \\
\hline $\mathrm{EBE} / \mathrm{CIN}$ & 10 & 1 & 9 & 90 & 10 & 7 & 3 & 30 & 0.031 \\
\hline SB/CIN & 10 & 5 & 5 & 50 & 10 & 7 & 3 & 30 & 0.500 \\
\hline
\end{tabular}




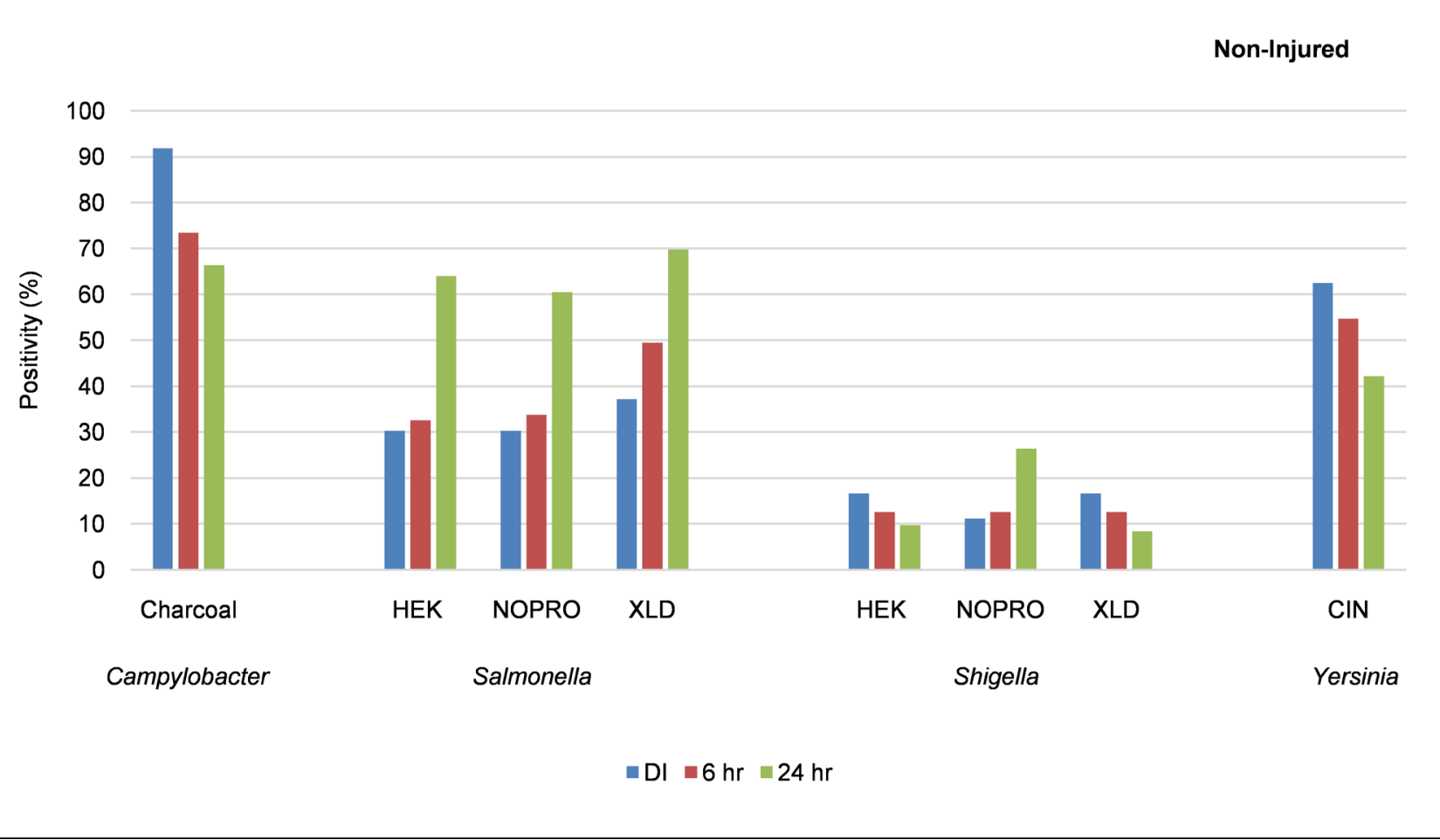

(a)

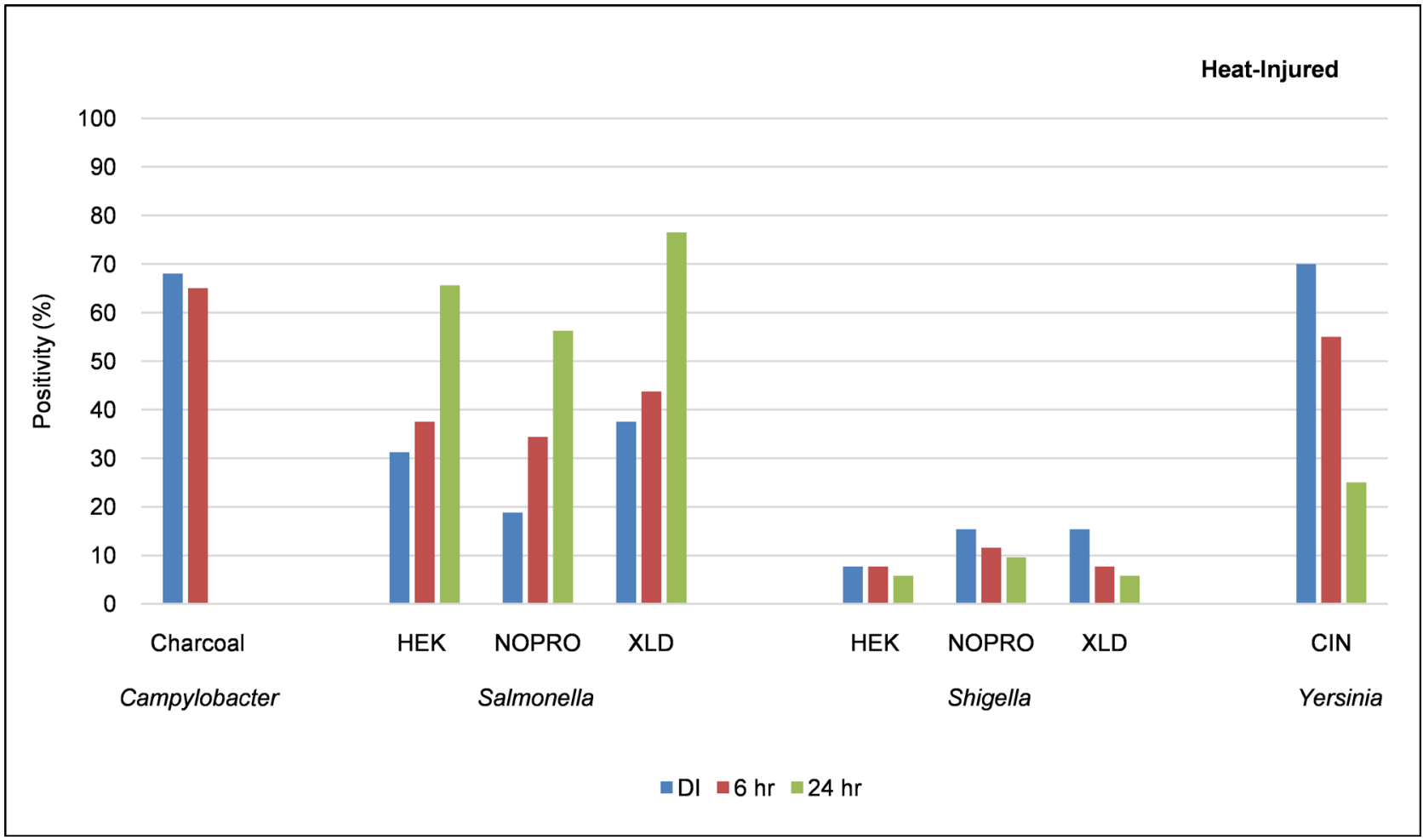

(b)

Figure 1. Comparison between percent recovery of isolates by media type for each enteric bacterial pathogen based on inoculation time point for all dilutions, non-injured (a) and heat injured (b). Abbreviations: Direct inoculation (DI); Hektoen agar (HEK); HardyChrom SS NoPro (NOPRO); Xylose Lysine Deoxycholate (XLD) agar; Cefsulodin, Irgasan, Novobiocin (CIN) agar. 


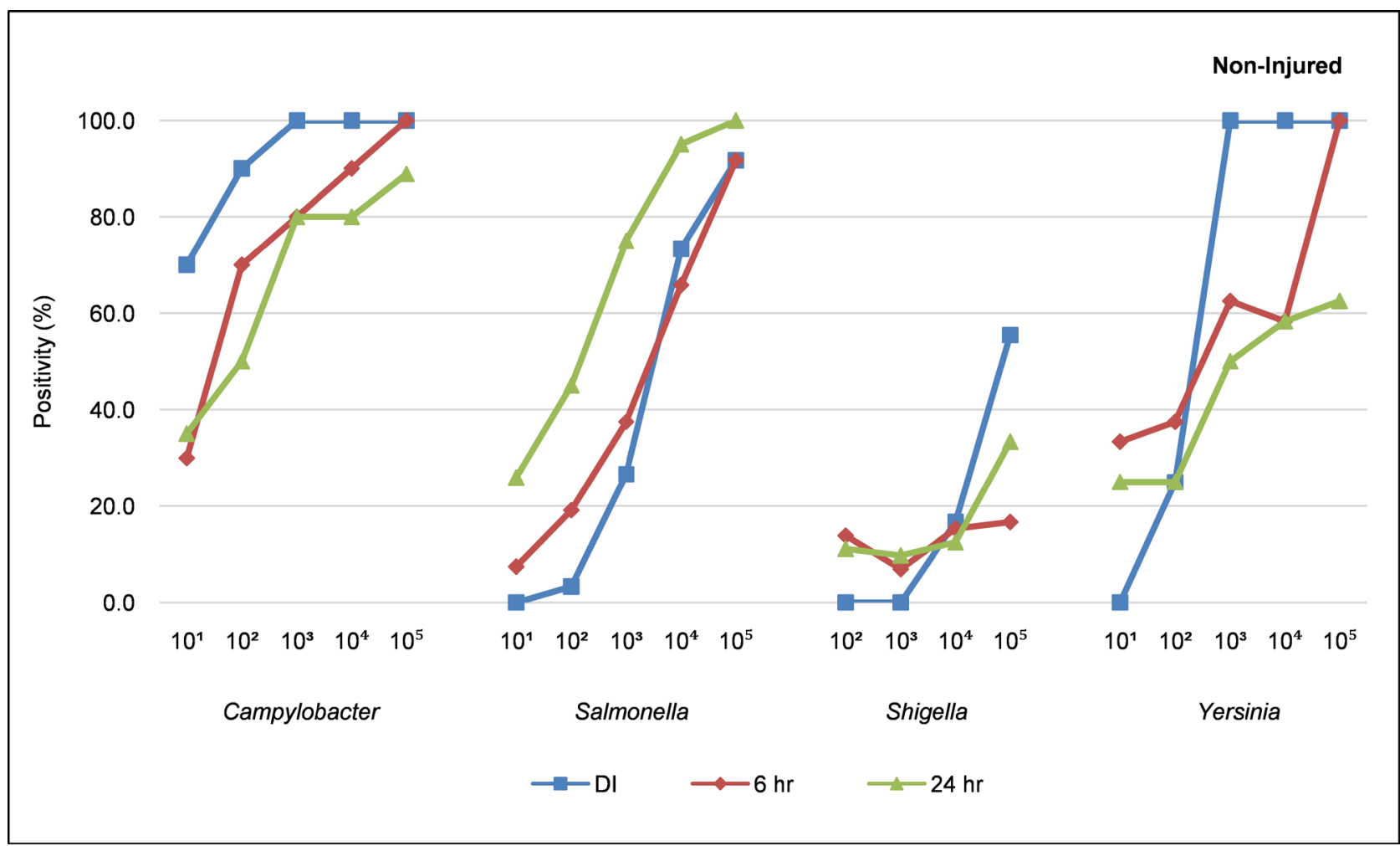

(a)

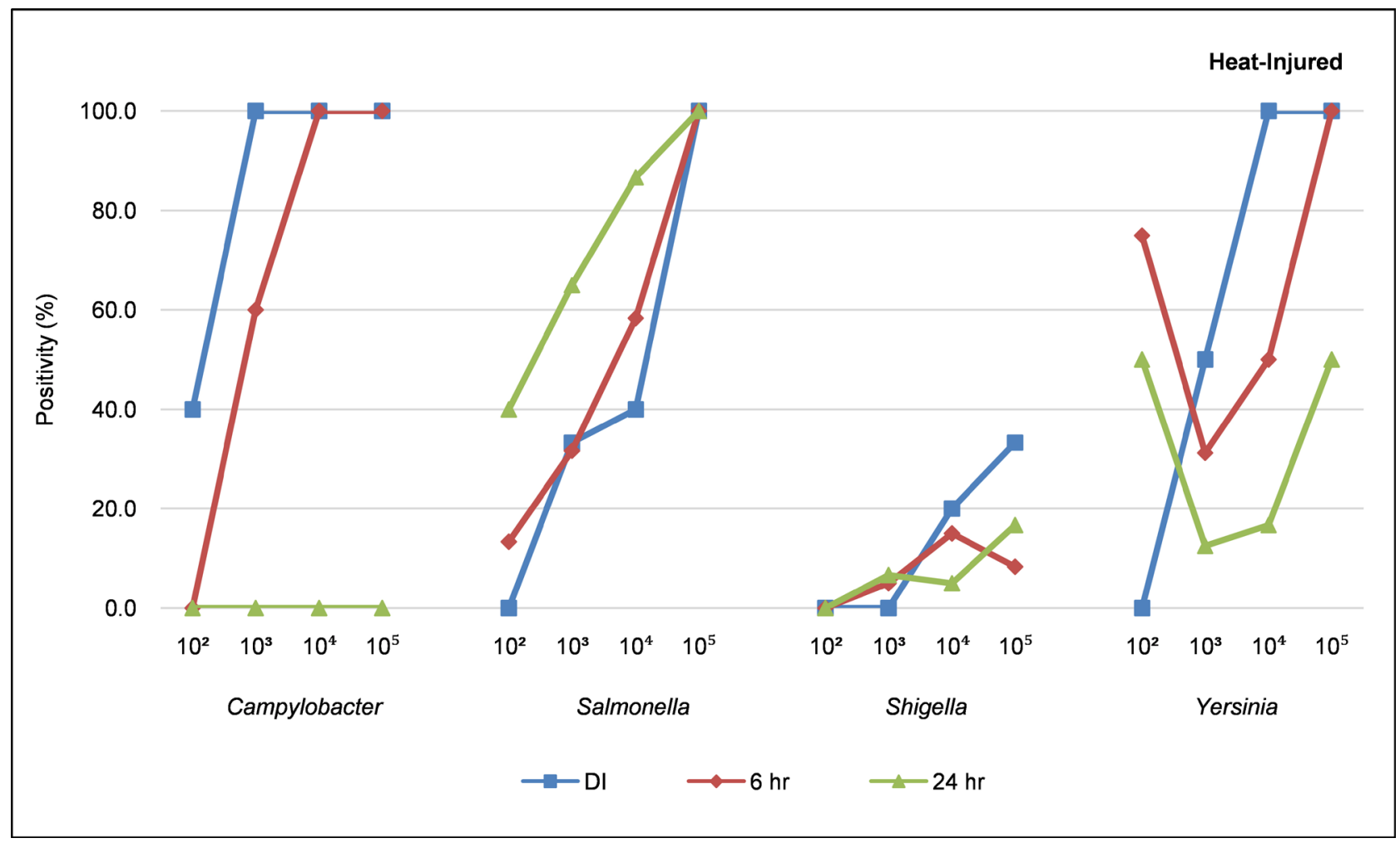

(b)

Figure 2. Isolation percentage based on each enteric bacterial pathogen stool dilution at direct inoculation (DI) and enrichment time points for (a) non-injured and (b) heat injured stools. 


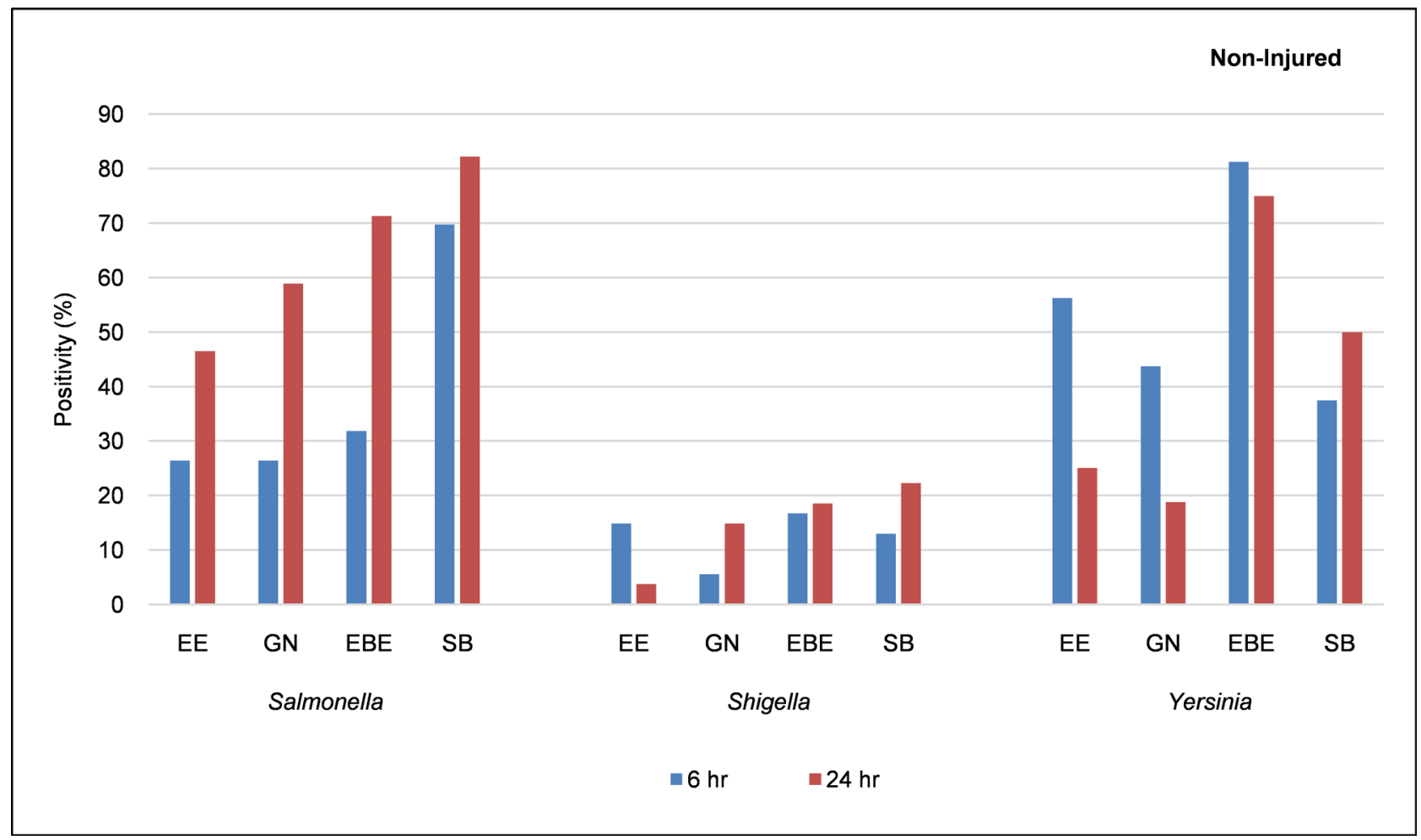

(a)

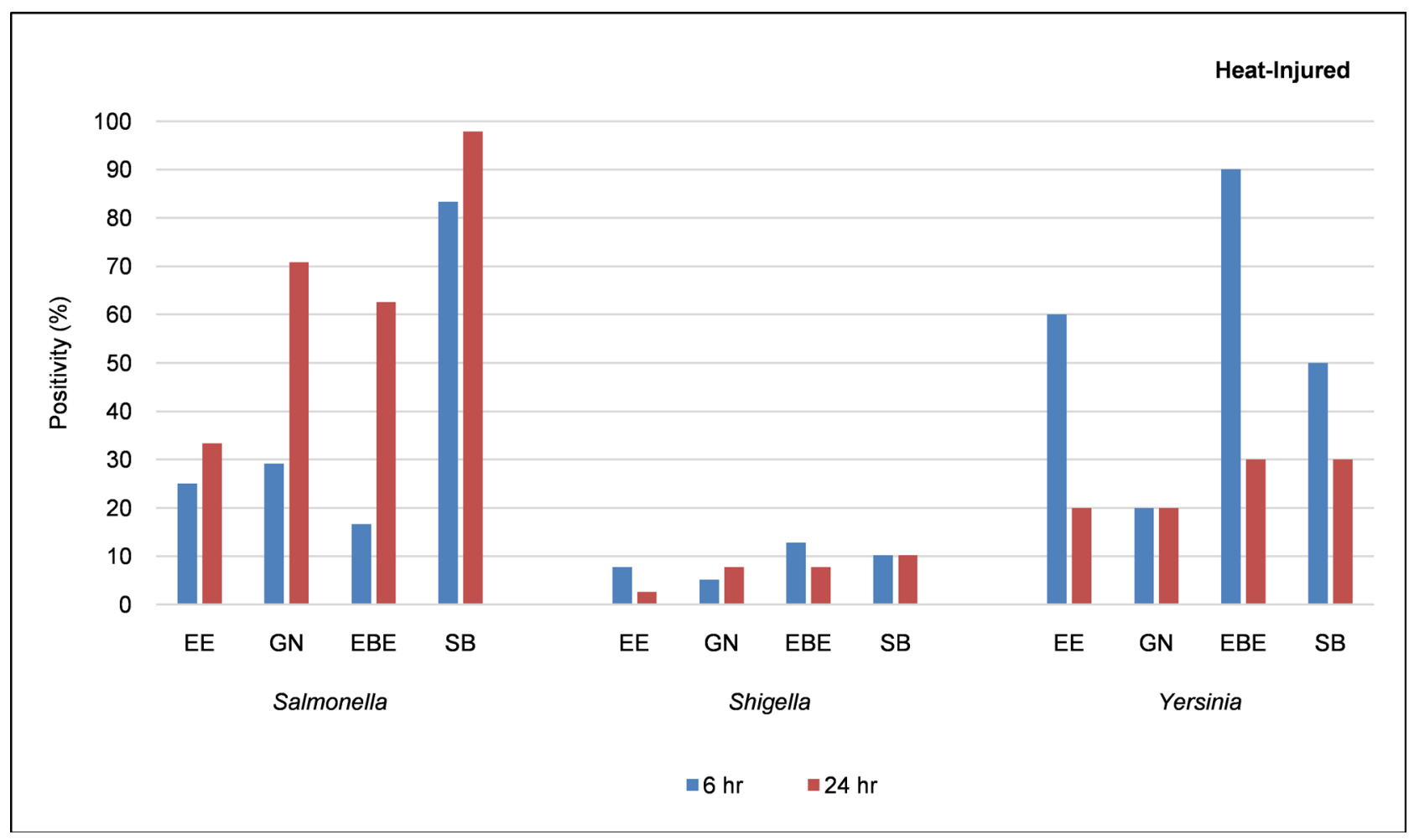

(b)

Figure 3. Comparison of isolates recovered by enrichment broth for Salmonella, Shigella, and Yersinia based on inoculation time point for all dilutions, non-injured (a) and heat injured (b). Abbreviations: EE Mossel (EE) broth; GN broth (GN); Enteric Bacterial Enrichment (EBE) broth; Selenite broth (SB). 
Direct inoculation of Yersinia stools was better than enrichment, although not significant (63\% for DI vs. $55 \%$ for 6 -hr and $42 \%$ for 24 -hr). Recovery with 6-hour enrichment outperformed 24-hour enrichment, $p=0.039$, Figure 1(a)). Bacterial concentrations were consistently recovered with direct inoculation down to $10^{3} \mathrm{CFU} / \mathrm{mL}$, but isolation of lower loads was better with 6-hour enrichment, although based on small sample size (Figure 2(a)). EBE broth performed much better than other broths in recovering Yersinia isolates $(81 \%$ at 6 -hr and $75 \%$ at 24 -hr, vs. $19 \%$ to $56 \%$ for other broths and subculture times, $\mathrm{p}<$ 0.001) (Figure 3(a)).

\subsection{Heat Injured Stools}

We also examined the potential consequences of extreme heat conditions that may result during transport. Generally, heat injured stool recovery was poor compared to non-heat injured with a higher limit of detection across organisms. A total of 59 stool specimens were setup and analyzed. Dilutions from $10^{5}$ to $10^{2}$ $\mathrm{CFU} / \mathrm{mL}$ were inoculated and subcultured. The organism stool distribution was as follows: Campylobacter $(\mathrm{n}=20)$, Salmonella $(\mathrm{n}=16)$, Shigella $(\mathrm{n}=13)$ and Yersinia $(\mathrm{n}=10)$. Briefly, recovery was lower for direct inoculation and after 24-hour enrichment for heat injured stools compared to non-heat injured stool specimens. At each enrichment subculture, every organism except Salmonella had significantly lower isolation than non-heat injured samples $(\mathrm{p}<0.05)$. Media and enrichment broth isolation trends were similar to non-injured stool data (Figures 1(b)-3(b) and Table 2). There was significant overgrowth of commensal flora on the heat injured 24-hour enrichment stool cultures which did not allow for identification of any Campylobacter isolates.

\section{Discussion}

In this study, our first step was to develop a broad-use enrichment for the cultivation of Campylobacter, Salmonella, Shigella, and Yersina termed Enteric Bacteria Enrichment (EBE) broth. Due in-part to the variety of growth requirements and incubation conditions unique to GI bacterial pathogens there is no routine multi-pathogen broth in clinical microbiology [2]. EBE broth was developed for incubation at $37^{\circ} \mathrm{C}$ with a mineral-oil overlay. Although, inconsistent with PHL role, we decided to use $37^{\circ} \mathrm{C}$ as our standard incubation temperature to establish a baseline for broth performance as if an unknown specimen was received at a sentinel laboratory for stool culture. The oil layer did not pose a recovery issue for the other facultative anaerobes [21]. In preliminary experiments, we observed no difference in Campylobacter isolation with the mineral oil overlay and incubation in a microaerophilic container (data not shown). Although, this testing was not exhaustive, and incubation at $42^{\circ} \mathrm{C}$ or the addition of supplemental antibiotics such as polymyxin $\mathrm{B}$ could increase recovery, this issue was not investigated [22].

Salmonella and Shigella are common organisms submitted to PHL for con- 
firmation and characterization. We compared organism recovery of two gold-standard agars (HEK and XLD) with a recently released chromogenic agar for Salmonella and Shigella (SS NoPro). XLD performed the best for Salmonella, and Shigella isolation was similar across media types except for SS NoPro after 24-hour enrichment. XLD agar has been documented as superior to other gold-standard broad-use enteric agars for recovery of these organisms [15] [23] [24]. For Salmonella and Shigella enrichment, SB and EBE were comparable. Optimal agar/broth combinations varied for Salmonella and Shigella. SB/XLD was the best combination for Salmonella while SB and EBE coupled with SS NoPro were better for Shigella strains. Shigella stools were evaluated a magnitude higher than the rest of the spiked specimens due to an early indication that recovery was poor at lower dilutions.

CIN agar is routinely used in clinical diagnostics for Yersinia but there may have been a benefit to inoculating Yersinia strains to HEK and XLD for a comparison of broad-media use as well [24] [25]. Gold-standard cold enrichment even performed on selective media, is time-consuming and labor intensive requiring multiple steps and is poorly suited for recovery of all Yersinia strains [9]. Nevertheless, incubation of EBE broth at $37^{\circ} \mathrm{C}$ may not allow for suitable Yersinia detection since the optimal enrichment temperature is maximally $25^{\circ} \mathrm{C}$ $30^{\circ} \mathrm{C}$. Most enterobacteria will outgrow Yersinia at the higher incubation temperature leading to underestimation.

This study is the first to our knowledge to evaluate enteric bacterial pathogen isolation from stool using a broad-use laboratory developed enrichment broth with standardized incubation conditions. Agar media supporting multiple enteric pathogens has been developed and evaluated for use with stool specimens [15] [18] [24]. The focus of enteric enrichment broth development has been mainly aimed at the recovery of a particular pathogen or simultaneous enrichment of multiple bacteria from food products [12] [20] [26] [27] [28] [29]. A universal pre-enrichment broth (UPB) was developed to enhance injured Gram-positive and Gram-negative enteric pathogen recover in food [29] [30]. UPB is non-selective making it difficult for use with specimens that have high levels of commensal flora. Multi-pathogen enrichment broths have been reported for isolation of organisms from food. For instance, the Selective Enrichment Broth (SEL) was formulated to allow the simultaneous growth of Salmonella enterica, E. coli O157:H7 and Listeria monocytogenes [12] [28]. Xiao et al. (2010) developed an enrichment broth to support simultaneous growth of Salmonella, Vibrio parahaemolyticus, and Vibrio cholerae [26]. The most promising multiplex enrichment broth effort was the development of the SSSLE broth supporting Salmonella, Staphylococcus aureus, Shigella flexneri, Listeria monocytogenes, and E. coli O157:H7 recovery from beef and pork [27]. Our study contained a wider variance of enteric bacterial strains and was conducted with human stool specimens opposed to food matrices.

There are some limitations to this study. Artificial stool specimens and heat 
stress conditions were contrived based on previous studies comparing enteric organism enrichment and isolation methods [28] [31] [32] [33] [34]. Routinely, stool specimens prior to PHL receipt would be stored by the sentinel laboratory before shipping or transportation to the PHL for processing decreasing bacterial concentration within the sample. This study did not account for storage or transport time prior to agar media or enrichment broth inoculation, thus recovery rates may be over-estimates of actual PHL observations. Organism distribution was skewed towards Campylobacter and Salmonella, although this is consistent with identifications seen clinically in the region. Initial EBE broth development plans included Vibrio and Shiga-toxin producing Escherichia coli O157 but no clinical isolates were available at our medical center for testing. Additionally, the workflow for Shiga-toxin producing E. coli confirmation can be labor and resource intensive including the reliance on a screening immunoassay prior to plating and isolate identification [35]. Future directions should include a clinical or public health laboratory based study involving true patient specimens and a larger more inclusive sampling. A comparison of EBE broth to standard practices can be more thoroughly conducted and optimal culture conditions can be established.

\section{Conclusion}

In conclusion, these findings have important implications related to bacterial enteric disease laboratory surveillance. With increased burden of pathogen isolation at PHL, procedural labor and resource costs strain laboratory infrastructure increases the need to provide efficient isolation methods. This study formulated an enrichment broth, EBE, which allowed the concurrent growth of multiple GI pathogens with standardized incubation. Our results suggest that there may only be utility for enrichment of CIDT positive Salmonella stool specimens. The data also indicate how difficult it is to successfully formulate a broth able to support a variety of bacterial GI pathogens. Based on our study data, it may be beneficial for PHL to eliminate direct inoculation of CIDT positive Salmonella specimens only pursuing enrichment and subsequent subculturing at a specific time-point for optimal fiscal and isolation balance. Enrichment of other GI bacterial pathogens may lack adequate recovery to balance associated labor and resources costs. Further exploration of enhancing culture yields and cost analyses for CIDT positive specimens is warranted.

\section{Conflicts of Interest}

The views expressed in this manuscript are those of the author(s) and do not reflect the official policy or position of the Department of the Army, Department of Defense, or the US Government. There are no conflicts of interest to disclose.

\section{References}

[1] Binnicker, M.J. (2015) Multiplex Molecular Panels for Diagnosis of Gastrointestinal 
Infection: Performance, Result Interpretation, and Cost-Effectiveness. Journal of Clinical Microbiology, 53, 3723-3728. https://doi.org/10.1128/JCM.02103-15

[2] Humphries, R.M. and Linscott, A.J. (2015) Practice Guidance for Clinical Microbiology Laboratories: Diagnosis of Bacterial Gastroenteritis. Clinical Microbiology Reviews, 28, 3-31. https://doi.org/10.1128/CMR.00073-14

[3] Langley, G., Besser, J., Iwamoto, M., Lessa, F.C., Cronquist, A., Skoff, T.H., Chaves, S., Boxrud, D., Pinner, R.W. and Harrison, L.H. (2015) Effect of Culture-Independent Diagnostic Tests on Future Emerging Infections Program Surveillance. Emerging Infectious Diseases, 21, 1582-1588. https://doi.org/10.3201/eid2109.150570

[4] Iwamoto, M., Huang, J.Y., Cronquist, A.B., Medus, C., Hurd, S., Zansky, S., Dunn, J., Woron, A.M., Oosmanally, N., Griffin, P.M. and Besser, J. (2015) Bacterial Enteric Infections Detected by Culture-Independent Diagnostic Tests-FoodNet, United States, 2012-2014. Morbidity and Mortality Weekly Report, 64, 252-257.

[5] Shea, S., Kubota, K.A., Maguire, H., Gladbach, S., Woron, A., Atkinson-Dunn, R., Couturier, M.R. and Miller, M.B. (2017) Clinical Microbiology Laboratories' Adoption of Culture-Independent Diagnostic Tests Is a Threat to Foodborne-Disease Surveillance in the United States. Journal of Clinical Microbiology, 55, 10-19. https://doi.org/10.1128/JCM.01624-16

[6] Imdad, A., Retzer, F., Thomas, L.S., McMillian, M., Garman, K., Rebeiro, P.F., Deppen, S.A., Dunn, J.R., Woron, A.M. (2018) Impact of Culture-Independent Diagnostic Testing on Recovery of Enteric Bacterial Infections. Clinical Infectious Diseases, 66, 1892-1898. https://doi.org/10.1093/cid/cix1128

[7] Ruiz, J., Nunez, M.L., Lorente, I., Perez, J., Simarro, E. and Gomez, J. (1996) Performance of Six Culture Media for Isolation of Salmonella Species from Stool Samples. European Journal of Clinical Microbiology and Infectious Diseases, 15, 922-926. https://doi.org/10.1007/BF01690509

[8] Nye, K.J., Fallon, D., Frodsham, D., Gee, B., Graham, C., Howe, S., Messer, S., Turner, T. and Warren, R.E. (2002) An Evaluation of the Performance of XLD, DCA, MLCB, and ABC Agars as Direct Plating Media for the Isolation of Salmonella Enterica from Faeces. Journal of Clinical Pathology, 55, 286-288.

https://doi.org/10.1136/jcp.55.4.286

[9] Savin, C., Leclercq, A. and Carniel, E. (2012) Evaluation of a Single Procedure Allowing the Isolation of Enteropathogenic Yersinia along with Other Bacterial Enteropathogens from Human Stools. PLoS ONE, 7, e41176. https://doi.org/10.1371/journal.pone.0041176

[10] Srijan, A., Wongstitwilairoong, B., Bodhidatta, L. and Mason, C. (2015) Efficiency of Plating Media and Enrichment Broths for Isolating Salmonella Species from Human Stool Samples: A Comparison Study. Open Journal of Medical Microbiology, 5, 231-236. https://doi.org/10.4236/ojmm.2015.54028

[11] Martiny, D., Dediste, A., Anglade, C., Vlaes, L., Moens, C., Mohamed, S. and Vandenberg, O. (2016) Performance of the Chrom ID Salmonella Elite Chromogenic Agar in Comparison with CHROMagar ${ }^{\mathrm{Tm}}$ Salmonella, Oxoid ${ }^{\mathrm{Tm}}$ Brilliance $^{\mathrm{Tm}}$ Salmonella and Hektoen Agars for the Isolation of Salmonella from Stool Specimens. Diagnostic Microbiology and Infectious Disease, 86, 128-130. https://doi.org/10.1016/j.diagmicrobio.2016.07.021

[12] Kim, H. and Bhunia, A.K. (2008) SEL, a Selective Enrichment Broth for Simultaneous Growth of Salmonella enterica, Escherichia coli O157: H7, and Listeria monocytogenes. Applied and Environmental Microbiology, 74, 4853-4866. https://doi.org/10.1128/AEM.02756-07 
[13] Gharst, G., Oyarzabal, O.A. and Hussain, S.K. (2013) Review of Current Methodologies to Isolate and Identify Campylobacter spp. from Foods. Journal of Microbiological Methods, 95, 84-92. https://doi.org/10.1016/j.mimet.2013.07.014

[14] Banwart, G.J. and Ayres, J.C. (1953) Effect of Various Enrichment Broths and Selective Agars upon the Growth of Several Species of Salmonella. Applied Microbiology, 1, 296-301. https://doi.org/10.1128/AM.1.6.296-301.1953

[15] Taylor, W.I. and Schelhart, D. (1971) Isolation of Shigellae: VIII. Comparison of Xylose Lysine Deoxycholate Agar, Hektoen Enteric Agar, Salmonella-Shigella Agar, and Eosin Methylene Blue Agar with Stool Specimens. Applied Microbiology, 21, 32-37. https://doi.org/10.1128/AM.21.1.32-37.1971

[16] Schiemann, D.A. (1979) Synthesis of a Selective Agar Medium for Yersinia enterocolitica. Canadian Journal of Microbiology, 25, 1298-1304.

https://doi.org/10.1139/m79-205

[17] Fitzgerald, C., Patrick, M., Gonzalez, A., Akin, J., Polage, C.R., Wymore, K., Gillim-Ross, L., Xavier, K., Sadlowski, J., Monahan, J. and Hurd, S. (2016) Multicenter Evaluation of Clinical Diagnostic Methods for Detection and Isolation of Campylobacter spp. from Stool. Journal of Clinical Microbiology, 54, 1209-1215. https://doi.org/10.1128/JCM.01925-15

[18] Perry, J.D. (2017) A Decade of Development of Chromogenic Culture Media for Clinical Microbiology in an Era of Molecular Diagnostics. Clinical Microbiology Reviews, 30, 449-479. https://doi.org/10.1128/CMR.00097-16

[19] Lue, Y.A. (1986) Is Enrichment Broth Necessary for Stool Cultures? Clinical Microbiology Newsletter, 8, 5-6. https://doi.org/10.1016/0196-4399(86)90097-8

[20] Hayashi, M., Kubota-Hayashi, S., Natori, T., Mizuno, T., Miyata, M., Yoshida, S., Zhang, J., Kawamoto, K., Ohkusu, K., Makino, S. and Ezaki, T. (2013) Use of Blood-Free Enrichment Broth in the Development of a Rapid Protocol to Detect Campylobacter in Twenty-Five Grams of Chicken Meat. International Journal of Food Microbiology, 163, 41-46. https://doi.org/10.1016/j.ijfoodmicro.2013.02.007

[21] Line, J.E. and Pearson, K.G. (2003) Development of a Selective Broth Medium for the Detection of Injured Campylobacter jejuni by Capacitance Monitoring. Journal of Food Protection, 66, 1752-1755. https://doi.org/10.4315/0362-028X-66.10.1752

[22] Fitzgerald, C. (2015) Campylobacter. Clinics in Laboratory Medicine, 35, 289-298. https://doi.org/10.1016/j.cll.2015.03.001

[23] Altwegg, M., Buser, J. and von Graevenitz, A. (1996) Stool Cultures for Shigella spp: Improved Specificity by Using MacConkey Agar with Xylose. Diagnostic Microbiology and Infectious Disease, 24, 121-124. https://doi.org/10.1016/0732-8893(96)00021-1

[24] Blom, M., Meyer, A., Gerner-Smidt, P., Gaarslev, K. and Espersen, F. (1999) Evaluation of Statens Serum Institut Enteric Medium for Detection of Enteric Pathogens. Journal of Clinical Microbiology, 37, 2312-2316. https://doi.org/10.1128/JCM.37.7.2312-2316.1999

[25] Shoaib, M., Shehzad, A., Raza, H., Niazi, S., Khan, I.M., Akhtar, W., Safdar, W. and Wang, Z. (2019) A Comprehensive Review on the Prevalence, Pathogenesis and Detection of Yersinia Enterocolitica. RSC Advances, 9, 41010-41021. https://doi.org/10.1039/C9RA06988G

[26] Xiao, X.L., Li, Y.J., Qin, Y.Y., Yu, Y.G. and Wu, H. (2010) A Multipathogen Selective Enrichment Broth for Simultaneous Growth of Salmonella spp., Vibrio parahaemolyticus, and Vibrio cholerae. Journal of General and Applied Microbiology, 56, 465-474. https://doi.org/10.2323/jgam.56.465 
[27] Chen, J., Tang, J., Bhunia, A.K., Tang, C., Wang, C. and Shi, H. (2015) Development of a Multi-Pathogen Enrichment Broth for Simultaneous Growth of five Common Foodborne Pathogens. Journal of General and Applied Microbiology, 61, 224-231. https://doi.org/10.2323/jgam.61.224

[28] Suo, B. and Wang, Y. (2013) Evaluation of a Multiplex Selective Enrichment Broth SEL for Simultaneous Detection of Injured Salmonella, Escherichia Coli O157: H7 and Listeria Monocytogenes. Brazilian Journal of Microbiology, 44, 737-742. https://doi.org/10.1590/S1517-83822013000300011

[29] Zhao, T. and Doyle, M. P. (2001) Evaluation of Universal Preenrichment Broth for Growth of Heat-Injured Pathogens. Journal of Food Protection, 64, 1751-1755. https://doi.org/10.4315/0362-028X-64.11.1751

[30] Bailey, J. S. and Cox, N. A. (1992) Universal Pre-Enrichment Broth for the Simultaneous Detection of Salmonella and Listeria in Foods. Journal of Food Protection, 55, 256-259. https://doi.org/10.4315/0362-028X-55.4.256

[31] Kamisaki-Horikoshi, N., Okada, Y., Takeshita, K., Sameshima, T., Kawasaki, S., Kawamoto, S. and Fratamico, P.M. (2011) Evaluation of TA10 Broth for Recovery of Heat-and Freeze-Injured Salmonella from Beef. Journal of AOAC International, 94, 857-862. https://doi.org/10.1093/jaoac/94.3.857

[32] Taylor, N., Gaur, R.L., Baron, E.J. and Banaei, N. (2012) Can a Simple Flotation Method Lower the Limit of Detection of Mycobacterium Tuberculosis in Extrapulmonary Samples Analyzed by the GeneXpert MTB/RIF Assay? Journal of Clinical Microbiology, 50, 2272-2276. https://doi.org/10.1128/JCM.01012-12

[33] Zheng, Q., Bustandi, C., Yang, Y., Schneider, K.R. and Yuk, H.G. (2013) Comparison of Enrichment Broths for the Recovery of Healthy and Heat-Injured Salmonella Typhimurium on Raw Duck Wings. Journal of Food Protection, 76, 1963-1968. https://doi.org/10.4315/0362-028X.JFP-13-041

[34] Saraka, N.D., Koffi, K.E., Koffi, K.S., Kouassi, K.S., Ehuie, P., Kette, F. and Dosso, M. (2014) Comparison of the Performances and Validation of Three Methods for Yersinia spp. Detection from Animals Stools Samples in Abidjan: A Non-Endemic and a Tropical Area. Journal of Applied Biosciences, 74, 6091-6098. https://doi.org/10.4314/jab.v74i1.13

[35] Gould, L.H. and STEC Clinical Laboratory Diagnostics Working Group (2012) Update: Recommendations for Diagnosis of Shiga Toxin-Producing Escherichia coli Infections by Clinical Laboratories. Clinical Microbiology Newsletter, 34, 75-83. https://doi.org/10.1016/j.clinmicnews.2012.04.004 